\title{
Dating the Hematoma of Haemorrhagic Stroke by Analysis of the disintegration of Oxyhemoglobin Nuclei
}

\author{
BIAOU Dimon Jean, ASSOGBA Kokou, VIANOU Antoine
}

\begin{abstract}
Hemorrhagic stroke is caused by the rupture of cerebral blood vessels discharging blood onto the surrounding tissues and caused the hematoma. The knowledge of the evolutionary profile of the hematoma is essential at the end of the stroke, because it allows specialists to direct the treatment. The present work aims to develop a method to characterize the four stages of evolution of the hematoma. The material consists of haemorrhagic CT scan images collected at the Cotonou CNHU CT scan unit and processed in MATLAB R2015a environment. The proposed method made it possible to determine a mathematical function that can model the density distribution of oxyhemoglobins in the hematoma. The results obtained made it possible to characterize the different evolutionary stages of the hemorrhagic stroke with a success rate of $97,09 \%$ on a database of 103 images. This method of dating the hematoma will certainly be a decision-making tool in the diagnosis and management of haemorrhagic stroke.
\end{abstract}

Index Terms - Clot, Evolutionary profile, hemorrhagic stroke, oxyhemoglobin

\section{INTRODUCTION}

The stroke is a neurological deficit suddenly caused by an infarction or hemorrhage. The hemorrhagic stroke, is caused by the rupture of cerebral blood vessels spilling blood into the surrounding tissues. The hematoma is the blood clot resulting from cerebral hemorrhage [2]. According to the WHO (2015) report on the causes of death worldwide, ischemia and hemorrhagic stroke are largely ahead of respiratory diseases, cancer and AIDS. WHO [6] does not hesitate to talk about Pandemic and expects a progressive increase in the incidence of stroke worldwide from 16 million cases in 2005 to nearly of 23 million in 2030. The strokes are nowadays a major public health problem.In general, in case of stroke, when the

Manuscript revised on September 17, 2019 and published on October 10,2019

BIAOU Dimon Jean, Ecole Doctorale Sciences de l'Ingénieur, Université d'Abomey-Calavi (UAC), Laboratoire d'Electrotechnique de Télécommunications et d'Informatique Appliquée (LETIA), 01 BP 2009 RP Cotonou, Bénin.

ASSOGBA Kokou, Ecole Doctorale Sciences de l'Ingénieur, Université d'Abomey-Calavi(UAC), Laboratoire d'Electrotechnique de Télécommunications et d'Informatique Appliquée (LETIA), 01 BP 2009 RP Cotonou, Bénin.

VIANOU Antoine, Ecole Doctorale Sciences de l'Ingénieur, Université d'Abomey-Calavi(UAC), Laboratoire d'Electrotechnique de Télécommunications et d'Informatique Appliquée (LETIA), 01 BP 2009 RP Cotonou, Bénin. life threatening is engaged, it is the cerebral CT scan without injection which is the examination most often carried out urgently for the diagnosis of hematoma [3]. The goal of imaging is to make the diagnosis of hematoma, is to know the evolutionary profile, and to recognize the underlying causes because of the risk of bleeding recurrence and treatment possibilities.

Table 1 : WHO statistics and forecasts (Source : [7])

\begin{tabular}{lll}
\hline YEAR & $\begin{array}{l}\text { Number of } \\
\text { Victims }\end{array}$ & $\begin{array}{l}\text { Number of } \\
\text { Deaths }\end{array}$ \\
\hline 1990 & 10100000 & 4700000 \\
\hline 2010 & 16800000 & 5900000 \\
\hline 2030 & 23000000 & 12000000 \\
\hline \hline
\end{tabular}

The precision in the diagnosis and the promptness in the care are essential at the stroke outcome. This is why the establishment of a method for dating the hematoma is certainly a decision-making tool that can provide more precision and speed in the diagnosis of hemorrhagic stroke. Four evolutionary profiles of the hematoma are classically distinguished: the hyper-acute, acute, subacute and chronic stages. For example, we have:

- In the hyper-acute stage (3 to 6 hours)

The clot consists of a heterogeneous mass composed of red blood cells filled with oxyhemoglobins. In CT scan, the hematoma is hyperdense compared to the cerebral parenchyma but it can be heterogeneous and contain hypodense areas [5], [2].

- $\quad$ At the sub-acute stage (3 days-4 weeks)

The oxidative denaturation of hemoglobin progresses and deoxyhemoglobin is transformed into methemoglobin. On CT scan, the density of the hematoma decreases further, the edges become blurred, isodense and hypodense [5], [2].

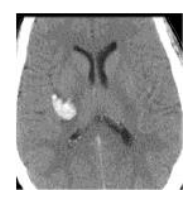

Hyperacute stage

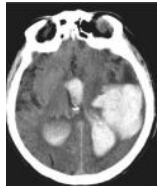

Acute stage

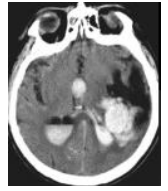

Sub-acute stage

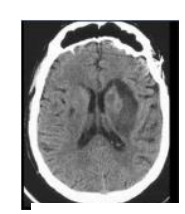

Chronic stage
Fig. 1. Different stages or Evolutionary

\section{MATERIAL AND METHODS}

\section{A. Material}

The material consists of brain CT scan images collected at the CT scan unit of the Hubert MAGA University Hospital 
Center (CNHU-HKM) located at the Faculty of Health Sciences of Cotonou and supplemented by images for free download on the Net. This image database was processed in a Matlab version 8.5 environment (R2015a)

\section{B. Methods}

1) Disintegration of oxyhemoglobin nuclei

The hematoma dating method used here is based on the knowledge of the anatomical transformations of the hematoma [1]. The global analysis of the hematoma transformations shows a continuous decrease of the oxyhemoglobin level in the hematoma. In fact, the density of oxyhemoglobins, generally represented in the image of the CT scan, by blocks of white pixels, gradually decreases from the edges of the hematoma towards the center following the evolutionary stages of the hemorrhagic stroke to cancel at the chronic phase [2]. This decrease in oxyhemoglobin level can be modeled by the radioactive decay function.

In a sample of radioactive material consisting of radioactive nuclei of a given species, the number of nuclei will decrease over time, and will be noted

$\mathrm{N}(\mathrm{t})$. If we call $\mathrm{N}_{0}$ the number of nuclei initially present, we have the relation :

$$
N(t)=N_{0} e^{-\lambda t}
$$

With $\lambda$ the probability that a kernel disintegrates per unit of time.

From the relation Eq. (1) we can determine $\lambda$

$$
\lambda=\frac{1}{t} \log \frac{N_{0}}{N(t)}
$$

The analysis of a database of brain CT scan images of the same individual over several days made it possible to draw an empirical curve of the decay of oxyhemoglobins.

\section{2) Determination of a reference threshold $T_{0}$}

The threshold $\mathrm{T}_{0}$ sought here corresponds to the threshold of the distributions of the oxyhemoglobins at the date $\mathrm{T}_{0}$ corresponding to the day 1 of the beginning of the stroke. To do this, the Otsu threshold determination method was applied to all day 1 images. The average of the calculated thresholds made it possible to obtain a reference threshold $\mathrm{T}_{0}$.

Otsu's [8] method automatically detects the threshold by seeking to minimize the variance within each region, which amounts to maximizing the variance between the two regions. This Otsu thresholding algorithm can be summed up to $[4]$ :

$T_{\text {otsu }}=\operatorname{argmin}_{T}\left\{\sum_{k<T} h(k)\left(\mu_{0}-\mu\right)^{2}+\sum_{K \geqq T} h(k)\left(\mu_{1}-\mu\right)^{2}\right\}$

With $: \mu=\operatorname{moy}\{\mathrm{I}(\mathrm{x})\}, \mu_{0}=\operatorname{moy}\{\mathrm{I}(\mathrm{x})$ for $\mathrm{I}(\mathrm{x})<\mathrm{T}\}$ and $\mu_{1}=$ $\operatorname{moy}\{\mathrm{I}(\mathrm{x})$ for $\mathrm{I}(\mathrm{x}) \geq \mathrm{T}\}$. $\mathrm{h}$ being the histogram function.

\section{3) Correlation between the empirical curve and the modeling function}

- Kolmogorov-Smirnov test

In order to determine the value of the parameter $\lambda$ which makes it possible to obtain a correlation between the empirical curve and the theoretical function modeling this distribution, the Komogorov-smirnov test is used.

Indeed, the Kolmogorov-Smirnov (KS) bilateral $D_{n}$ test statistic is widely used to measure the quality of fit between the empirical distribution of a set of $\mathrm{n}$ observations and a continuous probability distribution. It is defined by :

$$
D_{n}={ }_{x}^{\sup }\left|G(x)-\widehat{G}_{n}(x)\right|
$$

Where $n$ is the number of (independent) observations, $G_{n}$ is the function of the empirical cumulative distribution and $G$ is a fully specified continuous theoretical cumulative distribution function. Let $F_{n}$ be the cumulative distribution function of $\mathrm{D}_{\mathrm{n}}$ responding to the null hypothesis $\mathrm{H}_{0}$ for which the $n$ observations are independent and whose function is of cumulative distribution $\mathrm{G}$, we have :

$$
F_{n(x)}=P\left[\mathrm{D}_{\mathrm{n}} \leq x \mid H_{0}\right] \text { for } \mathrm{x} \in[0,1]
$$

The value $\lambda$ corresponding to the hypothesis $\mathrm{H}_{0}=0$ is then the correlation value of the empirical curve and the theoretical curve.

\section{The general algorithm of the method is:}

VARIABLES InputImage, SizeofImage, N0, landa, Dn, N(t), n PREDEFINED FUNCTIONS SmirnovTest ASSIGNMENT H0=1, landa $=0,009$

BEGING

//Détermination of No per size of image

READ InputImage

IF (SizeofImage $=114 \times 106$ ) THEN

$\mathrm{N} 0=9303$

WHILE (Dn !=0)

n++

DO

Landa $=$ landa- 0.001

$\mathrm{N}(\mathrm{t})=\mathrm{N} 0 * \exp (-$ landa $* \mathrm{t})$

END WHILE

ELSE IF (SizeofImage $=75 \times 96$ ) THEN

N0 $=2650$

WHILE (Dn !=0)

$\mathrm{n}++$

DO

Landa $=$ landa- 0.001

$\mathrm{N}(\mathrm{t})=\mathrm{N} 0 * \exp (-$ landa $* \mathrm{t})$

END WHILE

ELSE IF (SizeofImage $=34 \times 34$ ) THEN

$\mathrm{N} 0=90(3)$

WHILE(Dn !=0)

$\mathrm{n}++$

DO

Landa=landa-0.001

$\mathrm{N}(\mathrm{t})=\mathrm{N} 0 * \exp (-$ landa $* \mathrm{t})$

END WHILE

END IF

END

// Détermination of reference threshold

VARIABLES refInputImage, $\mathrm{n}, \mathrm{T} 0$ 
International Journal of Research in Advent Technology, Vol.7, No.9, September 2019

$$
\text { E-ISSN: 2321-9637 }
$$

Available online at www.ijrat.org

PREDEFINED FUNCTIONS OtsuThreshold BEGING

READ RefInputImage

$\mathrm{T}=$ OtsuThreshold(refInputImage)

FOR (1 to $n)$

DO

$\mathrm{T} 0=\sum(\mathrm{T}) / \mathrm{n}$

END FOR

END

\section{RESULTS}

\section{A. Disintegration equation of oxyhemoglobin nuclei}

Table 2 : Decay equation of oxyhemoglobins as a function of hematoma size

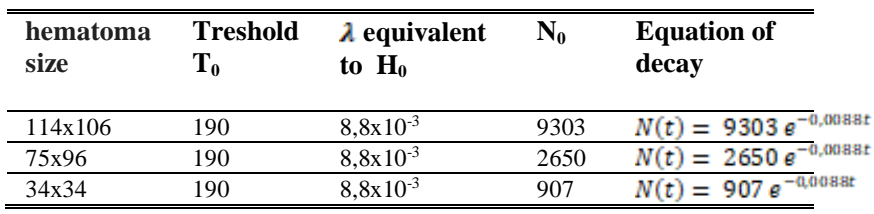

Correlation curve $\mathrm{H}_{0}=0$

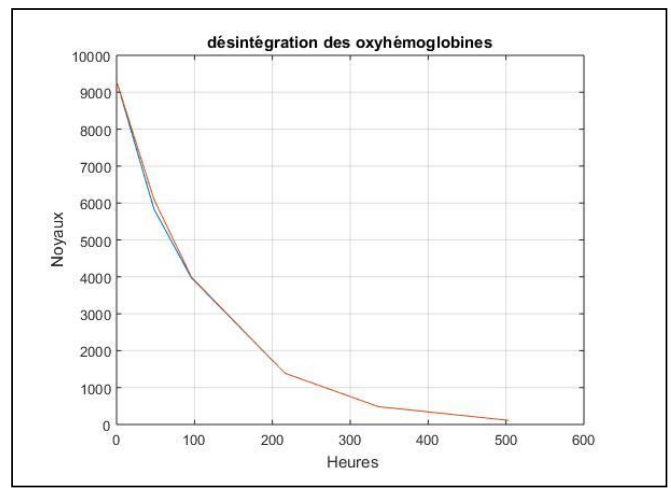

Fig. 2. Kolmogorov-Smirnov Test Correlation Curve

B. Hematoma dating result

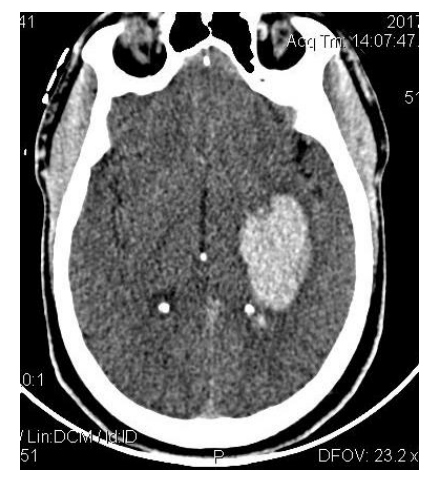

Fig. 3. CT1 hematoma dating curve

Datation CT $2 \mathrm{t}=\mathbf{8 0 , 3 5}$ hours, day 8 . Evolutionary profile : Subacute stage

\section{Hematoma dating result}

CT1
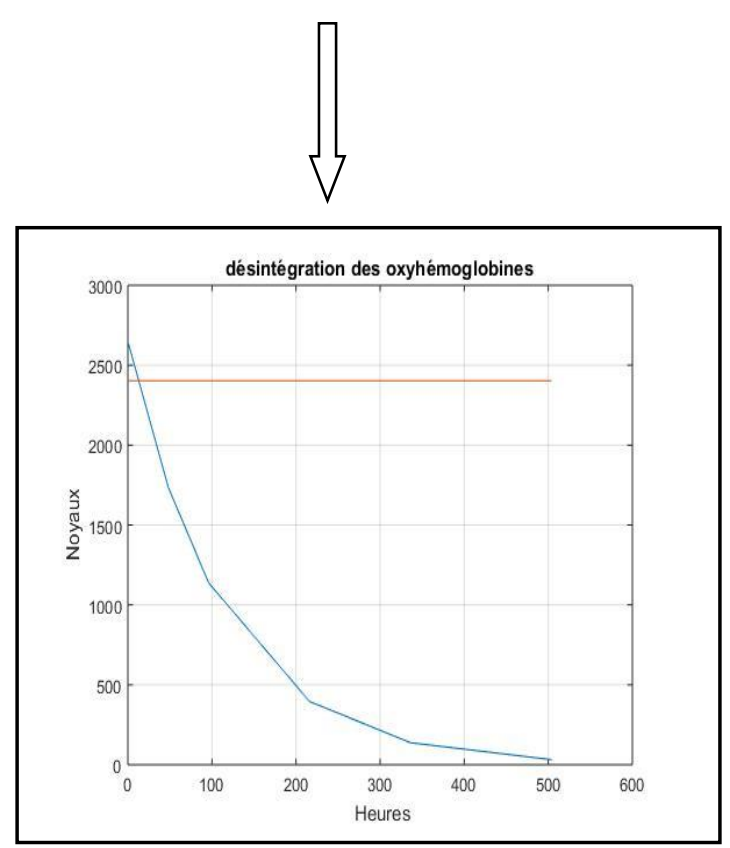

Fig. 4. CT1 hematoma dating curve

Dating CT1 $t=12,99$ hours, day 1

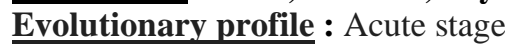

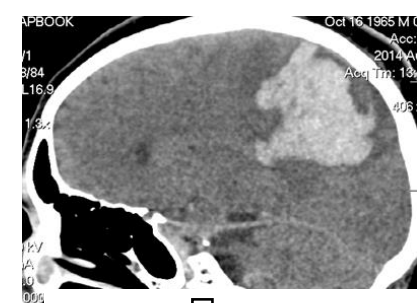

CT2
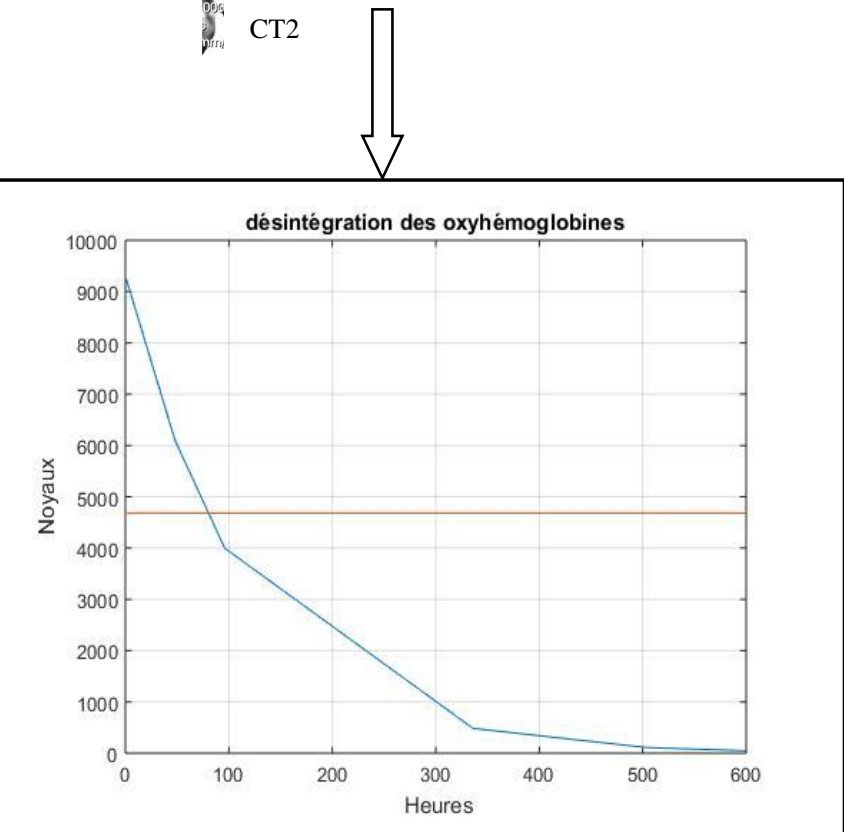
International Journal of Research in Advent Technology, Vol.7, No.9, September 2019

E-ISSN: 2321-9637

Available online at www.ijrat.org

\section{Summary of the application of the method on the images of the database}

Table 3 : Percentages of the application of the method

\begin{tabular}{lrr}
\hline & Numbers & Percentage \\
\hline Hyperacute & 4 & $3,88 \%$ \\
\hline Acute & 46 & $44,46 \%$ \\
\hline Subacute & 40 & $38,83 \%$ \\
\hline Chronic & 10 & $9,71 \%$ \\
\hline Success & 100 & $97,03 \%$ \\
\hline Failure & 3 & $2,91 \%$ \\
\hline Total & 103 & $100 \%$ \\
\hline
\end{tabular}

The relatively low failure rate demonstrates the effectiveness of the method in dating hematomas. Indeed this rate is related to the poor quality of some images including those download on the net.

\section{CONCLuSion}

In this study we proceeded to the characterization of hemorrhagic stroke by dating the evolutionary profile of the hematoma. Otsu's inter-mean algorithm allowed us to make a judicious choice of the reference threshold in order to determine the empirical decay curve. The Kolmogorov test made it possible to adjust the parameter $\lambda$ to obtain a perfect correlation $\left(\mathrm{H}_{0}=0\right)$ between the empirical curve and the theoretical curve. The results obtained made it possible to characterize the different evolutionary stages of the hemorrhagic stroke with a success rate of $97,09 \%$ on a database of 103 images. We can conclude that globally the atomic decay function has made it possible to model the distribution of the density of oxyhemoglobins in the hematoma and consequently to characterize the four evolutionary stages of the hemorrhagic stroke.

\section{REFERENCES}

[1]. Clark R.et al., 1990. Acute hematomas: effects of deoxygenation, hematocrit, and fibrin-clot formation and retraction on T2 shortening Radiology 1990; 175, 201-206.

[2]. Domitille M., 2015. Pathologie neurovasculaire aigue [en ligne] (2015) adresse url www.uclimaging.be/.../des.../des1_2015_2_pathologie_vasculaire_cer ebrale_aigue.pdf, consulté le 12/08/2017

[3]. JFR, 2014. Diagnostique et Interventionnelle, 62ème Journées Françaises de Radiologie Diagnostique et Interventionnelle. JFR 2014.

[4]. Krähenbühl A., 2014. Segmentation et analyse géométrique : application aux images tomodensitométriques de bois. Imagerie médicale. Université de Lorraine

[5]. Leclerc X. et al., 2003. Imagerie des hématomes intracérébraux non traumatiques, Journal of Neuroradiology $\mathrm{Vol} 30, \mathrm{~N}^{\circ}$ 5, 303-316

[6]. Marsaglia, G., W. Tsang, and J. Wang. "Evaluating Kolmogorov's Distribution." Journal of Statistical Software. Vol. 8, Issue 18, 2003

[7]. Mendis, S., Puska, P., Norrving, B., World Health Organization, World Heart Federation, World Stroke Organization, 2011. Global atlas on cardiovascular disease prevention and control. World Health Organization: World Heart Federation: World Stroke Organization, Geneva.

[8]. Otsu, N., 1979. "A Threshold Selection Method from Gray-Level Histograms," IEEE Transactions on Systems, Man, and Cybernetics, Vol. 9, No. 1, 62-66

\section{AUTHORS PROFILE}

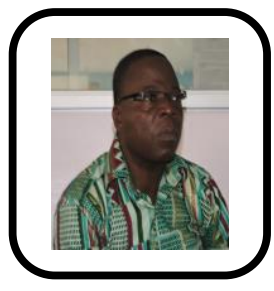

Dimon Jean BIAOU obtained his MASTER in Network and Telecommunications in 2013, and the Diplôme d'Etudes Approfondies (DEA) from the Graduate School of Engineering Sciences in 2015. He is currently a $\mathrm{PhD}$ student at the University of Abomey-Calavi (UAC) at the LETIA laboratory under the direction of Kokou Assogba. His research interests include Image processing applied to stroke, Artificial Intelligence, Object detection and tracking.

Dr. Kokou ASSOGBA obtained his PhD degree in Images and Signal Processing in 1999 at University Paris XII Val de Marne, France. He is working, since as teacher researcher in Computer Science and Electronics at University of Abomey-Calavi. He is Senior Member AND Head of the Laboratory of Electronics, Telecommunications and Applied nformatics (LETIA), he recently became Director General of Higher Education. His main research interests are: Digital Image processing, Pattern Recognition, Images Segmentation and smart technology.

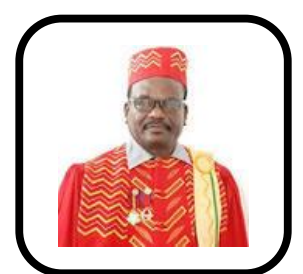

Pr. Antoine VIANOU, full Professor in Engineering Science and Technology, Vice-Chancellor of the University of Abomey-Calavi (UAC) Knight of the International Order of Academic Palms CAMES. Academician: Member of the National Academy of Sciences, Arts and Letters of Benin. President of the Sectorial Scientific Committee Engineering Sciences and Techniques / UAC Quality Assurance Consultant in Higher Education. He recently became Director of the UAC Graduate School of Engineering Sciences 